\title{
DVM Regimen
}

National Cancer Institute

\section{Source}

National Cancer Institute. DVM Regimen. NCI Thesaurus. Code C161963.

A chemotherapy regimen consisting of cisplatin, vindesine, and mitoguazone that may be used in the treatment of certain head and neck cancers. 